\title{
Trial Inclusion and Exclusion Criteria Supplemental Qualifiers Dataset
}

National Cancer Institute

\section{Source}

National Cancer Institute. Trial Inclusion and Exclusion Criteria Supplemental Qualifiers

Dataset. NCI Thesaurus. Code C147258.

A dataset containing supplemental information, specifically non-standard variables, to parent records in the trial inclusion/exclusion criteria domain. 